\title{
Übersicht
}

Notfall Rettungsmed 2020 $\cdot$ 23:529-537 https://doi.org/10.1007/s10049-020-00767-8 Online publiziert: 7 . September 2020

c) Springer Medizin Verlag $\mathrm{GmbH}$, ein Teil von Springer Nature 2020

\section{Video online}

Die Online-Version dieses Beitrags (https:// doi.org/10.1007/s10049-020-00767-8) enthält drei ergänzende Videos. Beitrag und Zusatzmaterial stehen Ihnen auf www. springermedizin.de zur Verfügung. Bitte geben Sie dort den Beitragstitel in die Suche ein, das Zusatzmaterial finden Sie beim Beitrag unter „Ergänzende Inhalte“.

\section{Einleitung}

Bei der Versorgung von Patienten in der zentralen Notaufnahme hat die Sonographie aufgrund ihrer einfachen, bettseitigen Anwendbarkeit einen großen Stellenwert. Unmittelbar nach Anamnese und körperlicher Untersuchung durchgeführt, ermöglicht sie auch bei Patienten mit kardiorespiratorischen Erkrankungen frühzeitig Entscheidungen über weitere diagnostische und erste therapeutisch notwendige Maßnahmen $[1,2]$.

So überrascht es nicht, dass auch im Rahmen der aktuellen COVID-19Pandemie bereits zahlreiche Studien und Erfahrungsberichte über Anwendung, Befunde und Stellenwert der Lungensonographie (LUS) bei Patienten mit dieser neuen Infektionskrankheit publiziert wurden [3-14].

Ziel dieses Übersichtsartikels ist die Präsentation typischer Ultraschallbefunde dieser Infektionskrankheit und die Diskussion ihres klinischen Stellenwerts.

G. Kunze ${ }^{1,3} \cdot$ E. Kovacikova' $\cdot$ H. Haller ${ }^{1}$ B. Kumle ${ }^{2,3}$

'Klinik für Innere Medizin I, Schwarzwald-Baar Klinikum Villingen-Schwenningen GmbH, VillingenSchwenningen, Deutschland

${ }^{2}$ Klinik für Akut- und Notfallmedizin, Schwarzwald-Baar Klinikum Villingen-Schwenningen GmbH, Villingen-Schwenningen, Deutschland

${ }^{3}$ DRF-Luftrettungsstation Christoph 11, Villingen-Schwenningen, Deutschland

\section{Lungenultraschall bei Patienten mit SARS-CoV-2-Infektion}

\section{Grundlagen der Lungen- sonographie}

Aufgrund des Rippenthorax können nur ca. $70 \%$ der Lungenoberfläche sonographisch dargestellt werden. Durch die Luftfüllung der gesunden Lunge kommt es an der Lungenoberfläche zu einer Totalreflexion der Schallwellen mit Entstehung von Reverberationsartefakten, sog. A-Linien. Darüber hinaus finden sich auch vereinzelte vertikale Artefakte (sog. „B-Linien“), die vernachlässigbar sind, solange nicht mehr als 3-5 pro Schallfeld darstellbar sind. Weiterhin kann das typische Pleuragleiten sicher dargestellt werden. Pathologische Befunde an der Lunge können dann sonographisch erkannt werden, wenn der pathologische Befund an die Lungenoberfläche heran- reicht und dort interkostal darstellbar ist. $\mathrm{Zu}$ den darstellbaren Befunden gehören nach Mathis [15]:

- Extrapulmonale Befunde (Pleuraerguss, Pleuraempyem, Hämatothorax, pleurale Tumoren)

- Pneumothorax

- Veränderungen an der Pleura visceralis und parietalis und subpleurale Veränderungen (Pleuritis)

- Konsolidierungen (Lungenembolie, Pneumonien, Tumoren)

- Veränderungen des Lungeninterstitiums (deutliche Zunahme sog. B-Linien) und

- Veränderungen der Thoraxwand.

\begin{tabular}{|c|c|c|c|}
\hline & Konvexschallkopf & Linearschallkopf & Sektorschallkopf ${ }^{a}$ \\
\hline Frequenz (in MHz) & $2-5$ & $5-18$ & $1-4$ \\
\hline Eindringtiefe (in cm) & Bis 15 & 3-8 (frequenzabhängig) & Bis 20 \\
\hline Vorteile & "Allrounder" & Hohe Auflösung & Kleine Auflagefläche \\
\hline \multicolumn{4}{|l|}{ Geeignet bei } \\
\hline Pleuraerguss (mittel) & $\mathrm{Ja}$ & Ja & Ja \\
\hline Pleuraerguss (klein) & (Ja) & Ja & Nein \\
\hline Pneumothorax & Ja & Ja & Nein \\
\hline Pneumonie (mittel) & Ja & $\mathrm{Ja}$ & (Ja) \\
\hline Pneumonie (klein) & (Ja) & Ja & Nein \\
\hline Lungenembolie & Ja & Ja & Nein \\
\hline Pleuritis & (Ja) & Ja & Nein \\
\hline B-Linien & Ja & (Nein) & Ja \\
\hline Herz & $((\mathrm{Ja}))$ & Nein & Ja \\
\hline Intervention & Ja & Ja & $((\mathrm{Ja}))$ \\
\hline
\end{tabular}




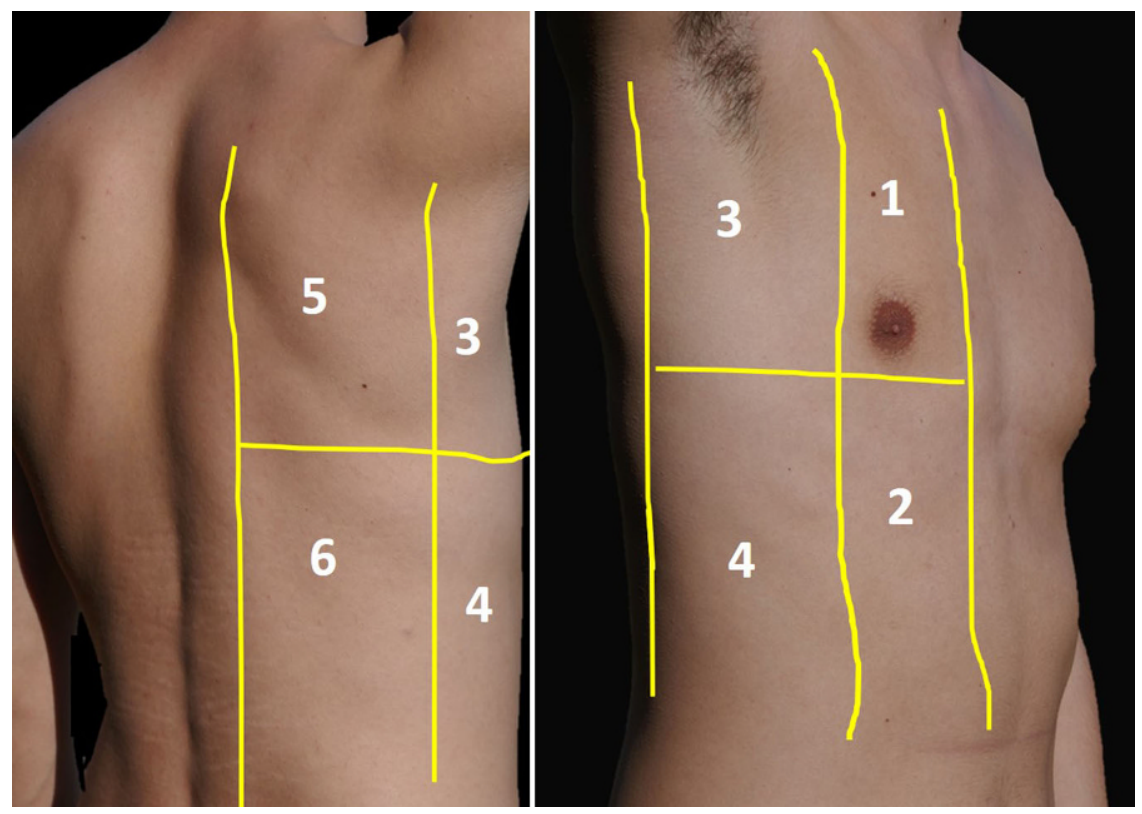

Abb. $1 \Delta$ Einteilung der Untersuchungsareale bei der Lungensonographie

\section{Histopathologische Befunde bei COVID-19}

Pathologisch werden bei COVID-19Pneumonien ein interstitielles Ödem, interstitielle entzündliche Infiltrate und gegebenenfalls auch ein intraalveoläres proteinreiches Exsudat beschrieben. Je nach Schwere der Erkrankung finden sich segmentale pneumonische Infiltrate bis hin zum diffusen Alveolarschaden mit hyalinen Membranen bei vorliegendem ARDS [16-24]. Weiterhin fallen makroskopisch plaqueartige Veränderungen auf der Pleura visceralis auf und selten eine fettige Proliferation derselben [25].

\section{Untersuchungstechnik und protektive Hygienemaßnahmen}

Die Ultraschalluntersuchung der Lunge wird üblicherweise mit multifrequenten Konvex- und Linearschallköpfen durchgeführt. Während sich mit Konvexschallköpfen sehr gut interstitielle Veränderungen (s. unten) und pneumonische Infiltrate darstellen lassen, können mit hochfrequenten Linearschallköpfen sehr gut die Pleura und die subpleurale Lunge untersucht werden. Diese hochauflösende Darstellung der Pleura ist technisch in vergleichbarer Auflösung und Qualität mit keinem anderen bild- gebenden Verfahren möglich. Für die Echokardiographie werden üblicherweise Sektorschallköpfe verwendet. Sofern ein anderes Preset verwendet wird, können mit Sektorschallköpfen auch größere pneumonische Infiltrate und ein interstitielles Syndrom dargestellt werden, die Darstellung kleiner (sub-)pleuraler Veränderungen ist mit Sektorschallköpfen nicht möglich. In der Praxis empfiehlt es sich, die Untersuchung mit dem Konvexschallkopf (alternativ Sektorschallkopf) zu beginnen und dann auf den Linearschallkopf zu wechseln. Der Farbdoppler kann hilfreich sein bei der Darstellung pulmonal- bzw. bronchialarterieller Gefäße in kleinen Konsolidierungen und kann dann eine Differenzierung zwischen Lungeninfarkten und entzündlichen Infiltraten ermöglichen. Vor- und Nachteile der verschiedenen Schallköpfe bei Anwendung zur Lungensonographie zeigt • Tab. 1.

Die deutschsprachigen Ultraschallfachgesellschaften sind übereingekommen, den menschlichen Thorax auf jeder Seite in 6 Regionen einzuteilen (• Abb. 1; [26]). Zur besseren Darstellung der Lunge sollte der Patient die Arme über den Kopf nehmen (Spreizen der Rippen), die Scapula kann durch Ergreifen der gegenüberliegenden Schulter "herausgedreht“ werden. Ventral und lateral kann sehr gut in Rückenlage un- tersucht werden, die Untersuchung der dorsalen Lunge gelingt am besten im Sitzen, weil so auch kleine Pleuraergüsse sicher detektiert werden können. Die vollständige Lungensonographie dauert bei entsprechender Expertise 5-15 min [10].

Da es sich bei der Sonografie um eine patientennahe Untersuchung handelt, muss der Untersucher bei Covid-19-Verdacht umfangreiche Schutzmaßnahmen (mindestens Kopfhaube, Schutzbrille und -kittel, 2 Paar Einmalhandschuhe) ergreifen. Zusätzlich sollte der Patient einen Mund-Nasen-Schutz tragen. Ultraschallgerät und Schallköpfe müssen nach der Untersuchung vollständig mit viruziden Tüchern gereinigt werden [27], dementsprechend bieten sog. „Kitteltaschengeräte" Vorteile. Bei Smartphone-/ Tablet-basierten Geräten muss darauf geachtet werden, dass deren Displays durch die notwendige Reinigung mit viruziden Tüchern keinen Schaden nehmen.

\section{Ultraschallbefunde bei SARS- CoV-2-Infektion}

Die sonographisch darstellbaren Befunde werden in 3 Kategorien eingeteilt:

a) Veränderungen der Pleura visceralis Durch eine entzündliche Alteration der Pleura und der subpleuralen Lunge erscheint die Oberflächenkontur der Pleura visceralis unregelmäßig und wirkt kurzstreckig unterbrochen bzw. „fragmentiert“. Die gegenüberliegende Pleura parietalis kann ebenfalls verdickt imponieren. Darüber hinaus findet sich wenig, ggf. gefangener Pleuraerguss (• Abb. 2, Video 1). Größere Pleuraergüsse sind bei COVID-19 sehr selten und müssen den Untersucher an andere Differenzialdiagnosen denken lassen.

b) Konsolidierungen

Die entzündliche, pneumonische Infiltration der Lunge mit flüssigkeitsgefüllten Alveolen führt zu einem solide, bisweilen leberähnlich imponierenden Lungenparenchym. Bisweilen findet sich im zuführenden Bronchus noch Luft im Sinne eines Bronchoaerogramms (- Abb. 3, Video 2). Wichtigste Differenzialdiagnose ist bei kleineren, 
dreiecksförmigen Konsolidierungen die Lungenembolie. Sofern mittels Beinvenensonographie kein Thrombosenachweis gelingt, hilft hier u. U. nur die Kontrastmittelsonographie bei der differenzialdiagnostischen Abklärung weiter. Während sich üblicherweise sowohl in großen als auch in kleinen pneumonischen Infiltraten farbdopplersonographisch Gefäße darstellen lassen, gibt es erste Hinweise, dass dies möglicherweise bei Infiltraten durch COVID-19 seltener der Fall ist [4].

c) Interstitielles Syndrom Im frühen Stadium zeichnet sich die COVID-19-Pneumonie durch ein interstitielles Ödem mit vermehrtem Nachweis (>3 pro Schallfeld) von sog. B-Linien aus. Bei Zunahme der interstitiellen Veränderungen verschmelzen diese B-Linien und die A-Linien verschwinden vollständig. Es ergibt sich das Bild einer „weißen Lunge“ (• Abb. 4, Video 3). Bei Nachweis eines interstitiellen Syndroms muss unterschieden werden, ob es sich um ein lokales Phänomen handelt oder ob weitere Areale der Lunge - ggf. auch symmetrisch - betroffen sind. Darüber hinaus müssen zahlreiche Differenzialdiagnosen bedacht werden (• Tab. 2; [28, 29]). Lange Zeit wurden die Begriffe „B-Linien“ und „Kometenschweifartefakte“ synonym verwendet. Heutzutage wird von „B-Linien“ im engeren Sinne nur dann gesprochen, wenn diese vertikalen Artefakte an der unauffälligen Pleuraoberflächenkontur entstehen. Entstehen sie an einer morphologisch veränderten Pleura (Konsolidierung oder Pleuritis), wird von Kometenschweifartefakten gesprochen. Da bei COVID-19 sowohl interstitielle als auch pleurale Veränderungen vorliegen, kommen beide Formen dieser vertikalen Artefakte vor. Für die bessere Darstellung dieser Artefakte und auch des Lungengleitens an sich ist es vorteilhaft, am Ultraschallgerät Artefaktunterdrückungsmechanismen (z.B. „Sono-CT“ u.a.) und Verfahren des sog. "post-proceeding“ auszuschalten. d) Verteilung der Veränderungen Die darstellbaren Veränderungen finden sich meistens bilateral und oft multifokal. Dabei sind basale, laterale und dorsale Lungenabschnitte häufiger betroffen als ventral und kranial gelegene Areale.

\section{Diskussion}

Bei der neuartigen SARS-CoV-2-Infektion wird zwischen einem asymptomatischen, einem milden, einem moderaten, einem schweren und einem kritischen Verlauf unterschieden (• Tab. 3). Während bezüglich der zur Klassifikation anzuwendenden klinischen Kriterien weitgehend Einigkeit herrscht, gibt es betreffend die erste anzuwendende Bildgebung unterschiedliche Stellungnahmen. So empfehlen die nordamerikanischen Fachgesellschaften, bei mildem Verlauf auf eine Bildgebung komplett zu verzichten, dagegen erfolgt in China die Triage zwischen mildem und moderatem Verlauf mithilfe der Bildgebung [30-35]. Als anzuwendendes bildgebendes Verfahren sprechen sich die nordamerikanischen Fachgesellschaften bei moderatem Verlauf für die Durchführung einer Röntgenübersichtsaufnahme aus, das chinesische National Health Institute äußert sich nicht dazu, welches Verfahren angewendet werden sollte.

In diesem Spannungsfeld der Frage, ob und mit welchem Verfahren eine ergänzende Bildgebung bei Patienten mit SARS-CoV-2-Infektion sinnvoll ist, bietet sich die Lungensonographie (LUS) als „Fortsetzung der klinischen Untersuchung mit technischen Mitteln“ [36] an.

Als zuverlässiges und kostengünstiges bildgebendes Verfahren kann es auch unter Einhaltung strikter Hygienemaßnahmen ubiquitär und bettseitig angewendet werden. Durch Reduktion klinikinterner Transporte infizierter Patienten in das CT kann weiterhin das Risiko einer Ansteckung des medizinischen Fachpersonals und anderer Patienten vermindert werden. Darüber hinaus kommt der Ultraschall ohne Strahlenbelastung mit der potenziellen Folge lebensbedrohender Krebserkrankungen im Langzeitverlauf aus [37, 38].
Notfall Rettungsmed 2020 $23: 529-537$ https://doi.org/10.1007/s10049-020-00767-8 (c) Springer Medizin Verlag GmbH, ein Teil von Springer Nature 2020

\section{G. Kunze · E. Kovacikova · H. Haller · B. Kumle}

\section{Lungenultraschall bei Patienten mit SARS-CoV-2- Infektion}

\section{Zusammenfassung}

Mithilfe des bettseitig, unmittelbar nach Anamnese und klinischer Untersuchung anwendbaren Lungenultraschalls können bei Patienten mit klinischen Zeichen eines Atemwegsinfekts und/oder respiratorischer Insuffizienz zahlreiche Ursachen und Komplikationen auf Anhieb erkannt werden. Damit ermöglicht der Lungenultraschall frühzeitig Entscheidungen über weitere diagnostische und erste therapeutische Maßnahmen auch bei Patienten, die sich mit V.a. SARS-CoV-2-Infektion in der zentralen Notaufnahme vorstellen. In diesem Übersichtsartikel werden die typischen lungensonographischen Befunde bei Patienten mit COVID-19 aufgezeigt und der Stellenwert der Sonographie verglichen mit anderen bildgebenden Verfahren bei dieser neuartigen Erkrankung diskutiert.

Schlüsselwörter

COVID-19 · Lungenultraschall · Interstitielles Syndrom · Lungenkonsolidierung .

Notaufnahme · Viruspneumonie

\section{Lung ultrasound in patients with SARS-CoV-2 infection}

\section{Abstract}

After taking a patient's history and physical examination, lung ultrasound can immediately reveal numerous causes and complications in patients suffering from respiratory tract infection and/or dyspnea. It can thus facilitate decisions on further diagnostic and first therapeutic procedures, even in patients with a SARS-CoV-2 infection who present to the emergency room. This review article highlights the typical findings of lung ultrasound in patients with COVID19 and discusses its value compared to other imaging methods.

Keywords

COVID-19 - Pulmonary ultrasonography . Interstitial syndrome - Pulmonary consolidation - Emergency department . Viral pneumonia 


\section{Übersicht}

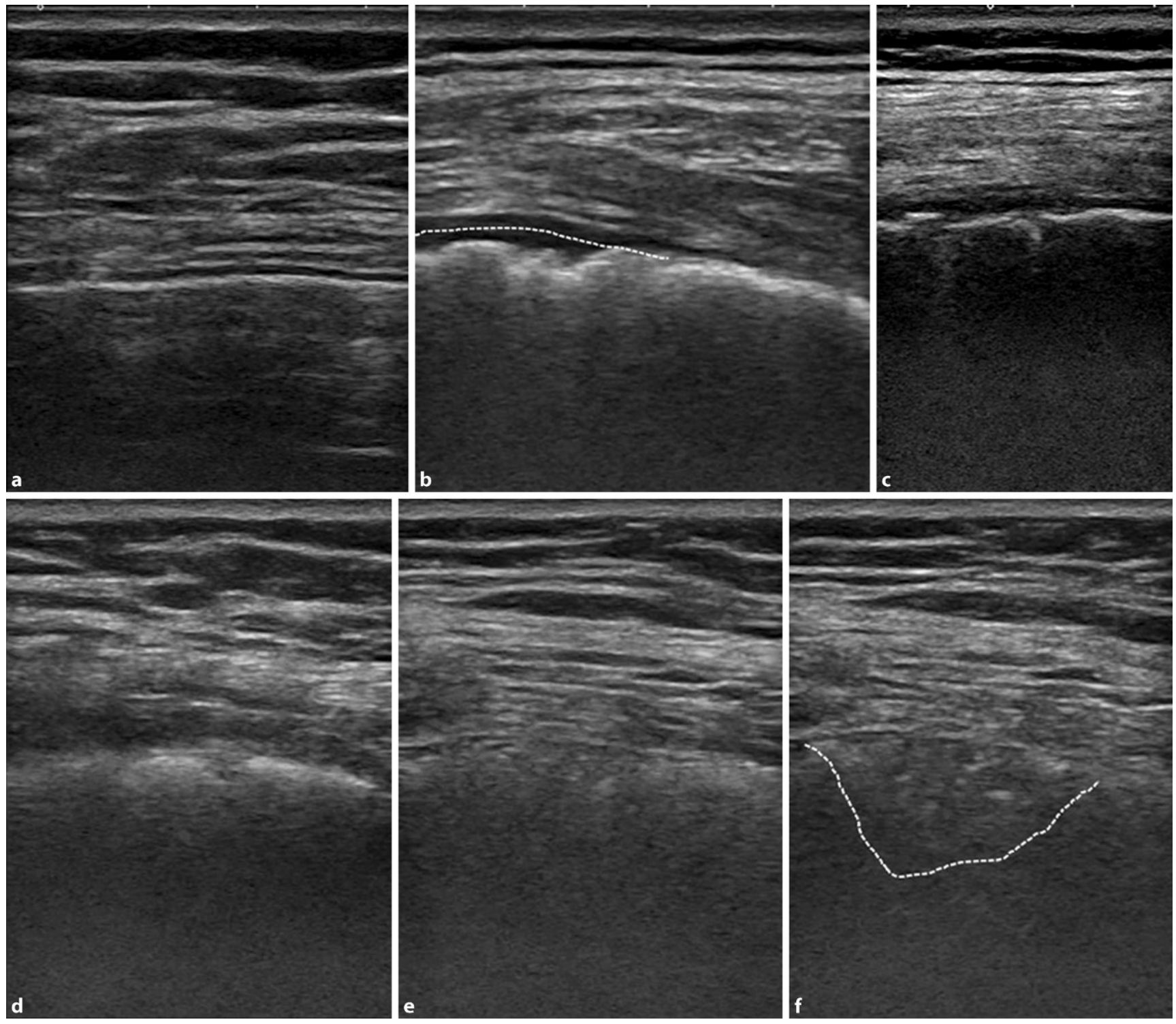

Abb. 2 \ Veränderungen der Pleura bei COVID-19, a Normalbefund, b lokale Verdickung der Pleura visceralis und parietalis ( gestrichelte Linie $=$ Pleuraspalt), $\mathbf{c}$ Fragmentierung der Pleura, $\mathbf{d}, \mathbf{e}$ verschiedene Stadien einer verwaschenen Pleura visceralis mit in $\mathrm{f}$ beginnender dreiecksförmiger Konsolidierung

Bei Patienten mit positivem PCRNachweis für COVID-19 zeigt sich als Ausdruck einer Pneumonie bei mildem bzw. moderatem Verlauf in der Mehrzahl der Fälle zunächst eine deutliche Zunahme von B-Linien an verschiedenen Arealen der Lunge (,interstitielles Syndrom“). Verschmelzende B-Linien (,weiße Lunge") mit Verschwinden der A-Linien und solide imponierende Infiltrate mit Nachweis eines Bronchoaerogramms sind Ausdruck schwergradiger pneumonischer Veränderungen $[4,7,8$, $11,39]$.
Nach eigenen Erfahrungen und ersten Studien zufolge eignet sich damit der LUS in der zentralen Notaufnahme zusammen mit Anamnese, Klinik, Labor und Blutgasanalyse sehr gut zur Einschätzung, welche COVID-19-Patienten ambulant geführt und welche stationär aufgenommen werden sollten [40]. Damit bietet sich der LUS auch sehr gut zur Klärung der Frage an, ob überhaupt eine ergänzende Thorax-CT indiziert ist. Weiterhin kann die Sonographie auch Hinweise auf wichtige Differenzialdiagnosen wie die Lungenembolie geben und diesen Verdacht nachfolgend mittels Echokar- diographie oder Beinvenensonographie bestätigen. Bei Thrombosenachweis erübrigt sich dann beispielsweise die ergänzende Durchführung einer Angio-CT, da unabhängig von deren Ergebnis eine harte Indikation zur Antikoagulation vorliegt [41]. Sofern bei Patienten mit sonographisch nachgewiesener Lungenembolie/Thrombose geklärt werden sollte, ob möglicherweise zusätzlich eine COVID19-Pneumonie vorliegen könnte, ist die Durchführung einer sog. Low-dose-CT ausreichend. 

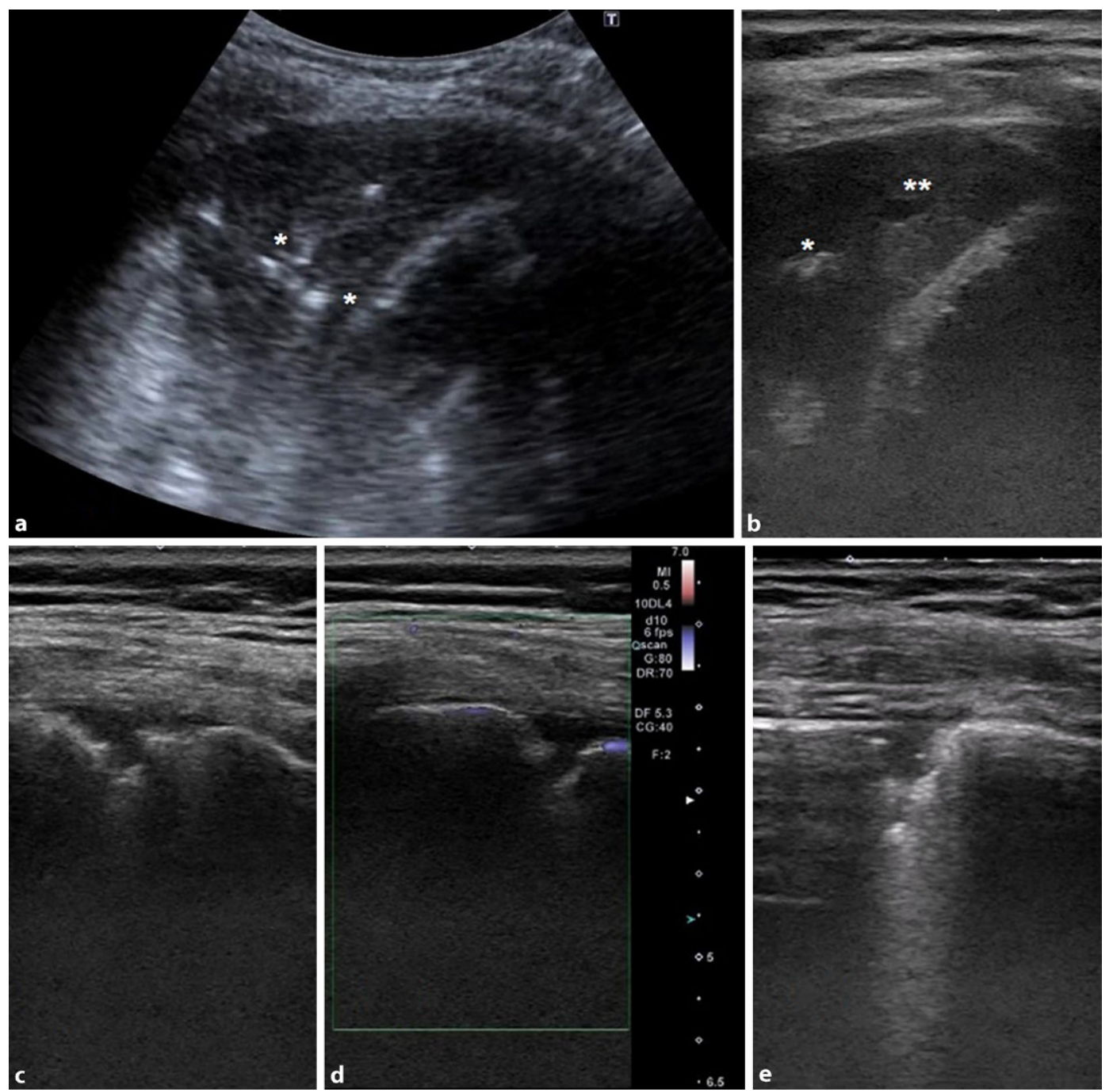

Abb. $3<$ Konsolidierungen bei COVID-19, a pneumonisches Infiltrat mit Bronchoaerogramm (weiße Reflexe), b dasselbe Infiltrat wie in a. Man erkennt mit dem hochauflösenden Schallkopf neben einem kleinen luftgefüllten ${ }^{*}$ ) auch einen sekretgefüllten ${ }^{* *}$ Bronchus, c kleine subpleurale Konsolidierung ohne Gefäßnachweis im Farbdoppler (d), was differenzialdiagnostisch an eine Konsolidierung bei Lungenembolie denken lässt, e kleine Konsolidierung bei COVID-19, sog. "Wasserfallzeichen"

\section{Differenzialdiagnosen und Stellenwert im Vergleich mit anderen bildgebenden Verfahren}

Studien, in denen zwischen dem Lungenultraschall und der Röntgenübersichtsaufnahme bei COVID-19 verglichen wurde, gibt es bislang nicht. Verglichen mit dem CT werden allerdings gerade bei moderatem Verlauf im Röntgenbild viele Infiltrate übersehen [42]. Die Computertomographie zeigt bei COVID-19Pneumonie dagegen nicht nur typische Befunde einer Viruspneumonie, sondern häufig auch ein für COVID-19 typisches Verteilungsmuster der Veränderungen [43-46]. Retrospektive Vergleichsstudien mit kleinen Fallzahlen zwischen CT und LUS lassen ahnen, dass die Schweregradeinteilung bei COVID-19-
Pneumonie in beiden Verfahren gut korreliert (• Tab. 4; $[4,10,11])$. Da nur $70 \%$ der Lungenoberfläche sonographisch darstellbar sind, können sonographisch zentrale und apikale Infiltrate nicht detektiert und somit eine pulmonale Manifestation bei bekannter SARS-CoV2-Infektion auch nicht ausgeschlossen werden. Allerdings ist bei fehlendem sonographischem Nachweis entsprechender Veränderungen allenfalls von einer milden pulmonalen Manifestation der Erkrankung auszugehen.

Bei Nachweis eines interstitiellen Syndroms sind zahlreiche Differenzialdiagnosen wie eine Herzinsuffizienz und Urämie zu berücksichtigen. Weiterhin ist die Aussage des LUS eingeschränkt bei Patienten mit vorbestehenden interstitiellen Lungenerkrankungen oder einer Lungenfibrose ( $\bullet$ Abb. 5). Hier dür- fen B-Linien und Kometenschweifartefakte allenfalls dann diagnostisch verwendet werden, wenn das Ausmaß dieser Veränderungen bei dem Patienten vorbekannt und es zu einer erheblichen Zunahme dieser Veränderungen gekommen ist. Auch wenn hierzu aussagefähige Vergleichsstudien fehlen, erscheint es naheliegend, dass bei diesen Patienten die Thorax-CT besser in der Lage ist, eine Viruspneumonie zu erkennen.

Fokal lokalisierte pulmonale und pleurale Veränderungen finden sich auch bei Pneumonien und Pleuritiden durch andere Erreger. Finden sich bei bislang gesunden Patienten jedoch alle o. g. typischen Kriterien in Kombination in verschiedenen Arealen, muss der Befund in der aktuellen Pandemiesituation als hoch suggestiv für das Vorliegen 


\section{Übersicht}
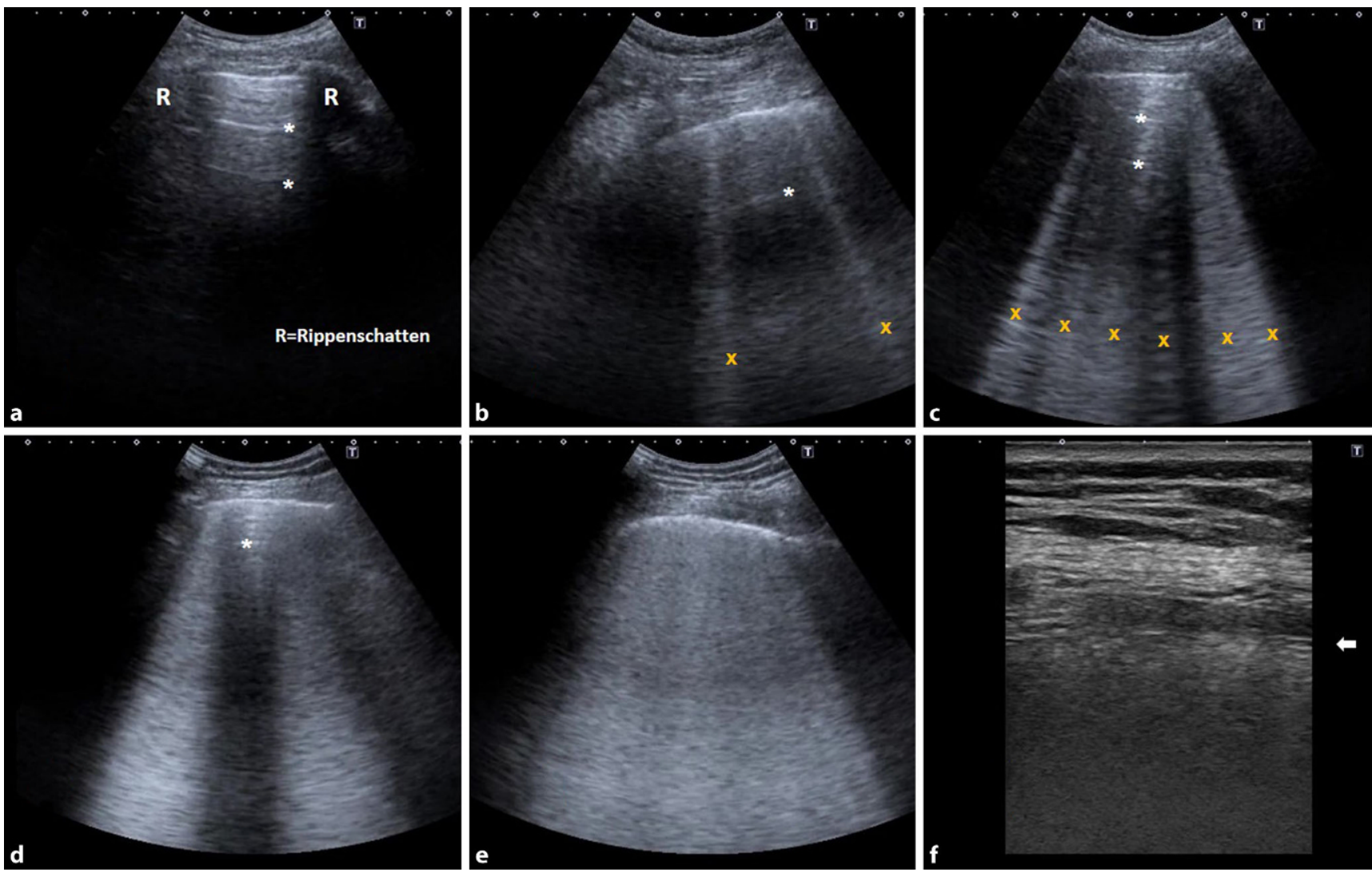

Abb. 4 A Interstitielles Syndrom bei COVID-19, a Normalbefund mit A-Linien $\left.{ }^{*}\right)$ und in b vereinzelten B-Linien $(x,<4 /$ Schallfeld). c Als Ausdruck eines interstitiellen Ödems findet sich bei einer COVID-19-Patientin eine deutliche Zunahme der B-Linien auf >3/Gesichtsfeld, die in d verschmelzen und zu einem zunehmenden Verschwinden der A-Linien $(*)$ führen. e Sog. „weiße Lunge“ durch verschmelzende B-Linien. An derselben Stelle findet sich in $\mathbf{f}$ mit dem hochauflösenden Schallkopf eine völlig verwaschene Pleura visceralis (Pfeil: Pleuraspalt)
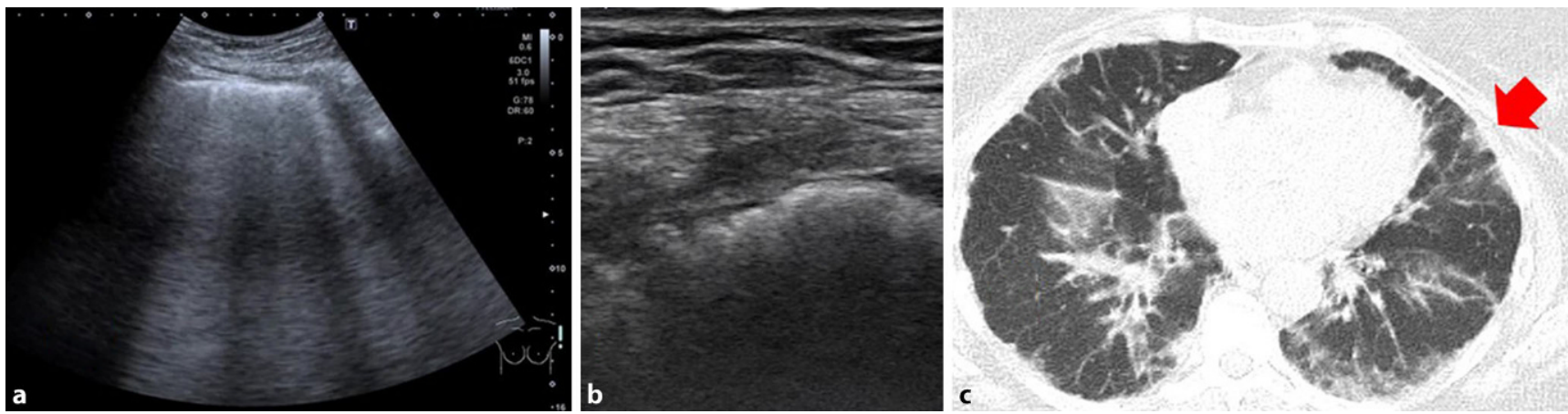

Abb. 5 A Differenzialdiagnose interstitielles Syndrom. 54-jährige Patientin mit bekannter Sarkoidose, die sich mit einem Infekt der oberen Atemwege in der Notaufnahme vorstellte. Sonographisch zeigt sich aufgrund der Sarkoidose ein ähnliches Bild wie bei einer COVID-19-Pneumonie mit verschmelzenden B-Linien (a) und kleinen unscharfen subpleuralen Konsolidierungen (b). Die im CT darstellbaren Veränderungen (c) wurden nicht als COVID-19-typisches Bild beschrieben. Das im CT mittels Pfeilmarkierte Areal entspricht den Ultraschallbildern in $\mathbf{a}$ und $\mathbf{b}$

einer COVID-19-Pneumonie angesehen werden.

\section{Bildgebung zum Screening bei Verdacht auf COVID-19}

In Phasen der Pandemie, als Notaufnahmen in Wuhan/China, in Italien und in den USA eine sehr große Anzahl von Patienten mit neu aufgetretenen
Atemwegsinfekten zu bewältigen hatten und gleichzeitig PCR-Tests nur begrenzt verfügbar und darüber hinaus mit Wartezeiten bis $\mathrm{zu} 30 \mathrm{~h}$ verbunden waren, wurde mangels Alternativen die Thorax-CT zum Screening auf Vorliegen einer möglichen SARS-CoV-2-Infekti- 
Tab. 2 Differenzialdiagnose interstitielles Syndrom $^{\mathrm{a}}$

\begin{tabular}{|ll}
\hline Lokal & Pleuritis \\
& Pneumonie \\
& Pleuranarben \\
& Lungenembolie \\
& Lungenkontusion \\
Multi- & Kardiale Stauung \\
fokal & Urämie \\
& ARDS \\
& Höhenlungenödem \\
& Interstitielle Lungenerkrankungen \\
& Lungenfibrose
\end{tabular}

Tab. 4 Punktescore bei Lungensonographie von COVID-19-Patienten ${ }^{a}$

\begin{tabular}{|ll}
\hline Befund & Punkte \\
\hline A-Linien, $<3$ B-Linien & 0 \\
\hline$>3$ B-Linien & 1 \\
\hline Konfluierende B-Linien & 2 \\
\hline Konsolidierungen & 3
\end{tabular}

on angewendet [47]. In entsprechenden Studien war die Sensitivität der CT zur Erkennung einer COVID-19-Pneumonie sogar höher als die der PCR im ersten durchgeführten Nasen-RachenAbstrich [48, 49]. Allerdings dürfen diese Daten, die über ein in der akuten Not geborenes Vorgehen gewonnen wurden, auf die aktuelle Situation im deutschsprachigen Raum mit beherrschbaren Infektionszahlen nicht einfach übertragen werden. Gerade in der frühen Phase der Erkrankung zeigt das CT sowohl bei asymptomatischen als auch bei symptomatischen Patienten in bis $\mathrm{zu} 56 \%$ einen Normalbefund [50-53] und die Befunde sind nicht spezifisch für eine COVID-19-Pneumonie [50, 54, 55]. Daher warnen mittlerweile radiologische Fachgesellschaften [56-58] weltweit vor dem Einsatz der CT als „screening tool“ zur Detektion einer SARS-CoV-2-Infektion und verweisen - auch in Hinblick
Tab. 3 Klinische Schweregradeinteilung bei SARS-CoV-2-Infektion. (Modifiziert nach [30-35])

\begin{tabular}{|c|c|c|c|c|}
\hline & Mild & Moderat & Schwer & Kritisch \\
\hline $\begin{array}{l}\text { Klinische Beschwerden eines Atemwegsinfekts } \\
\text { (Husten, Halsschmerzen, Fieber, Müdigkeit, } \\
\text { Kopf- und Gliederschmerzen) }\end{array}$ & $\mathrm{Ja}$ & $\mathrm{Ja}$ & $\mathrm{Ja}$ & $\mathrm{Ja}$ \\
\hline Atemnot (subjektiv) & Nein & Möglich & Ja & $\mathrm{Ja}$ \\
\hline Atemfrequenz (/min) & Normal & $>20$ & $>30$ & k. A. \\
\hline $\mathrm{O}_{2}$-Sättigung (Raumluft) (in \%) & $>93$ & $>93$ & $<93$ & $\ll 90$ \\
\hline Pneumonie & Nein & Ja & $\mathrm{Ja}$ & $\mathrm{Ja}$ \\
\hline Respiratorisches Versagen/Sepsis/MOV & Nein & Nein & Nein & Ja \\
\hline Management & Ambulant & Klinik & Klinik/IMC & Klinik/ICU \\
\hline
\end{tabular}

publizierte Triagealgorithmen müssen weiterhin kritisch unter Berücksichtigung der lokalen Pandemiesituation und der vorhandenen Infrastruktur geprüft werden [63].

Aktuell werden internationale Datenbanken und multizentrische Studien eingerichtet, die die verschiedenen bildgebenden Verfahren miteinander vergleichen [39, 64]. Mithilfe der daraus gewonnenen Erkenntnisse können hoffentlich zukünftig Algorithmen für eine optimierte, individuelle Patientenversorgung in verschiedenen Phasen der Pandemie erarbeitet werden.

\section{Fazit für die Praxis}

- Die Lungensonographie kann bettseitig unmittelbar nach Anamneseerhebung und klinischer Untersuchung als erstes bildgebendes Verfahren durchgeführt werden.

- Kleine, tragbare „Kitteltaschengeräte" sind besonders gut geeignet, da sie einfach und sicher desinfiziert werden können.

- Typische Befunde bei Patienten mit COVID-19-Pneumonie sind ein vermehrter Nachweis von B-Linien (,,interstitielles Syndrom"), entzündliche Veränderungen der Pleura und kleinere und größere Konsolidierungen mit und ohne Bronchoaerogramm.

- Der Befund eines „interstitiellen Syndroms" ist nicht spezifisch für eine Viruspneumonie und nur zusammen mit Anamnese und Klinik zu interpretieren.

- Verschmelzende B-Linien bis hin zur "weißen Lunge" und pneumonische Infiltrate zeigen schwergradigere Veränderungen an. 


\section{- Kleine dreiecksförmige Konsolidie- rungen lassen differenzialdiagnos- tisch an Lungenembolien denken. \\ - Veränderungen bei COVID-19 finden sich v. a. basal dorsal und lateral, seltener kranial und ventral.}

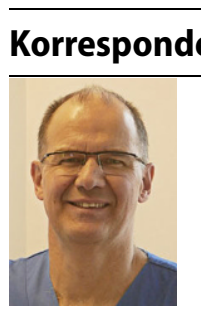

\section{enzadresse}

\section{Dr. med. G. Kunze}

Klinik für Innere Medizin I, Schwarzwald-Baar Klinikum Villingen-Schwenningen $\mathrm{GmbH}$

Klinikstr. 11, 78052 VillingenSchwenningen, Deutschland georg.kunze@sbk-vs.de

Danksagung. Die Autoren danken Dr. Frank Schmid vom Radiologischen Institut des Schwarzwald-Baar Klinikum Villingen-Schwenningen für die Bereitstellung der in diesem Artikel verwendeten CTBilder und die tägliche ausführliche Demonstration mit kritischer, konstruktiver und immer lehrreicher Diskussion der erhobenen Befunde.

\section{Einhaltung ethischer Richtlinien}

Interessenkonflikt. G. Kunze, E. Kovacikova, H. Haller und B. Kumle geben an, dass kein Interessenkonflikt besteht.

Für diesen Beitrag wurden von den Autoren keine Studien an Menschen oder Tieren durchgeführt. Für die aufgeführten Studien gelten die jeweils dort angegebenen ethischen Richtlinien. Für Bildmaterial oder anderweitige Angaben innerhalb des Manuskripts, über die Patienten zu identifizieren sind, liegt von innen und/oder ihren gesetzlichen Vertretern eine schriftliche Einwilligung vor.

\section{Literatur}

1. Blank W, Mathis G, Osterwalder J (2019) Kursbuch Notfallsonografie, 2. Aufl.

2. Rüttermann V (2020) Thoraxsonographie - eine wertvolle Ergänzung der Basisdiagnostik in der zeitnahen ambulanten Abklärung von Husten und Luftnot. Internist 61(1):13-20. https://doi.org/10. 1007/s00108-019-00721-y

3. Buonsenso D, Piano A, Raffaelli $F$ et al (2020) Point-of-Care Lung Ultrasound findings in novel coronavirus disease-19 pnemoniae. Eur Rev Med Pharmacol Sci 24(5):2776-2780. https://doi.org/ 10.26355/eurrev_202003_20549

4. Huang Y, Wang S, Liu Y et al (2020) A Preliminary Study on the Ultrasonic Manifestations of Peripulmonary Lesions of Non-Critical Novel Coronavirus Pneumonia (COVID-19). SSRN Journal. https://doi. org/10.2139/ssrn. 3544750

5. Mojoli F, Mongodi S, Orlando A et al (2020) Our recommendations for acute management of COVID-19. Crit Care 24(1):207. https://doi.org/10. 1186/s13054-020-02930-6
6. Piscaglia F, Stefanini F, Cantisani V et al (2020) Vorteile, offene Fragen und Herausforderungen des Einsatzes von Ultraschall in der Ära der COVID19-Pandemie. Die Ansichten einer Gruppe von internationalen Experten aus aller Welt. Ultraschall Med.https://doi.org/10.1055/a-1149-9872

7. Soldati G, Smargiassi A, Inchingolo Ret al (2020) Is there a role for lung ultrasound during the COVID19 pandemic? J Ultrasound Med. https://doi.org/ 10.1002/jum.15284

8. Soldati G, Smargiassi A, Inchingolo R et al (2020) On lung ultrasound patterns specificity in the management of COVID-19 patients. J Ultrasound Med. https://doi.org/10.1002/jum.15326

9. Vetrugno L, Bove T, Orso D et al (2020) Our Italian experience using lung ultrasound for identification, grading and serial follow-up of severity of lung involvement for management of patients with COVID-19. Echocardiography 37(4):625-627. https://doi.org/10.1111/echo. 14664

10. Lu W, Zhang S, Chen B et al (2020) Nichtinvasive Beurteilung von pulmonalen Läsionen bei Patienten mitCoronavirus-Erkrankung (COVID 19) durch Ultraschall direkt am Krankenbett. Ultraschall Med. https://doi.org/10.1055/a-11548795

11. Peng Q-Y, Wang X-T, Zhang L-N (2020) Findings of lung ultrasonography of novel corona virus pneumonia during the 2019-2020 epidemic. Intensive Care Med 46(5):849-850. https://doi. org/10.1007/s00134-020-05996-6

12. Höpfner M (2020) Covid-19 Thoraxsonographie. https://covid-sono.com/. Zugegriffen: 17. Mai 2020

13. Point-of-Care Ultrasound Certification Academy ${ }^{\text {TM }}$ Point-of-Care Ultrasound (POCUS) COVID-19 Video Resources. https://www.pocus.org/covid19-videos/.Zugegriffen: 17. Mai 2020

14. Kalafat $E$, Yaprak E, Cinar $G$ et al (2020) Lung ultrasound and computed tomographic findings in pregnant woman with COVID-19. Ultrasound Obstet Gynecol. https://doi.org/10.1002/uog. 22034

15. Mathis $\mathrm{G}$ (Hrsg) (2016) Bildatlas der Lungensonographie, 6. Aufl. Springer, Berlin, Heidelberg https://doi.org/10.1007/978-3-662-47536-2

16. Tian S, Hu W, Niu L et al (2020) Pulmonary pathology of early-phase 2019 novel Coronavirus (COVID-19) pneumonia in two patients with lung cancer. J Thorac Oncol 15(5):700-704. https://doi. org/10.1016/j.jtho.2020.02.010

17. Lacy JM, Brooks EG, Akers J et al (2020) COVID19. Am J Forensic Med Pathol. https://doi.org/10. 1097/PAF.0000000000000567

18. Karami P, Naghavi M, Feyzi A et al (2020) Mortality of a pregnant patient diagnosed with COVID-19. Travel Med Infect Dis. https://doi.org/10.1016/j. tmaid.2020.101665

19. Barton LM, Duval EJ, Stroberg E et al (2020) COVID19 autopsies, Oklahoma, USA. Am J Clin Pathol 153(6):725-733. https://doi.org/10.1093/AJCP/ AQAA062

20. Xu Z, Shi L, Wang Y et al (2020) Pathological findings of COVID-19 associated with acute respiratory distress syndrome. Lancet Respir Med 8(4):420-422. https://doi.org/10.1016/S22132600(20)30076-X

21. Menter T, Haslbauer JD, Nienhold R et al (2020) Post-mortem examination of COVID19 patients reveals diffuse alveolar damage with severe capillary congestion and variegated findings of lungs and other organs suggesting vascular dysfunction. Histopathology. https://doi.org/10. 1111/HIS.14134

22. Zeng Z, Xu L, Xie X-Y et al (2020) Pulmonary pathology of early phase COVID-19 pneumonia in a patient with a benign lung lesion. Histopathology. https://doi.org/10.1111/HIS.14138

23. Liu Q, Wang RS, Qu GQ et al (2020) Gross examination report of a COVID-19 death autopsy. Fa Yi Xue Za Zhi 36(1):21-23. https://doi.org/10. 12116/j.issn.1004-5619.2020.01.005

24. Smith MJ, Hayward SA, Innes SM et al (2020) Pointof-care lung ultrasound in patients with COVID19-a narrative review. Anaesthesia. https://doi. org/10.1111/anae.15082

25. Liu Q pers. Mitteilung zu Referenz Nr. 23

26. Stock K. https://www.degum.de/fileadmin/ dokumente/service/Downloads/Poster_A4Lungenultraschall-Protokoll_DEGUM_SGUM OEGM_V3_06042020_Print_digital_NEU.pdf? elqTrackld=6e5d8331362440b385ce7a8b431e 5 fc6\&elqaid=3969\&elqat=2. Zugegriffen: 17 . Mai 2020

27. Basseal J (2017) Guidelines for reprocessing ultrasound transducers. Aust J Ultrasound Med 20(1):30-40. https://doi.org/10.1002/ajum.12042

28. Yue Lee FC, Jenssen C, Dietrich CF (2018) A common misunderstanding in lung ultrasound. Med Ultrason 20(3):379-384. https://doi.org/10 11152/mu-1573

29. Soldati G, Demi M, Demi L (2019) Ultrasound patterns of pulmonary edema. Ann Transl Med 7(Suppl 1):16. https://doi.org/10.21037/atm.2019. 01.49

30. Dennie C, Hague C, Lim RS et al (2020) Canadian Association of Thoracic Radiology/Canadian Association of Radiologists Consensus Statement Regarding Chest Imaging in Suspected and Confirmed COVID-19. Can Assoc Radiol J. https:// doi.org/10.1177/0846537120924606

31. COVID-19TreatmentGuidelinesPanel.Coronavirus Diseases 2019 (COVID-19) Treatment Guidelines. https://www.covid19treatmentguidelines.nih. gov/.Zugegriffen:12. Mai 2020

32. American College of Radiology (2020) https:// www.acr.org/Advocacy-and-Economics/ACRPosition-Statements/Recommendations-forChest-Radiography-and-CT-for-SuspectedCOVID19-Infection.Zugegriffen: 17. Mai 2020

33. Centers for Disease Control and Prevention (2020) https://www.cdc.gov/coronavirus/2019-ncov/ hcp/clinical-guidance-management-patients. html\#lab-findings. Zugegriffen: 11. Mai 2020

34. National Health Commission of the People's Republic of China Diagnosis and treatment protocol for COVID-19 (trial version 7). http://en.nhc.gov.cn/ 2020-03/29/c_78469.htm. Zugegriffen: 17. Mai 2020

35. Gandhi RT, Lynch JB, Del Rio C (2020) Mild or moderate Covid-19. N Engl J Med. https://doi.org/ 10.1056/NEJMcp2009249

36. Seitz K (2016) In memoriam Professor Dr. med. Gerhard Rettenmaier 9. April 1929-7. Dezember 2015. Z Gastroenterol 54(2):177. https://doi.org/ 10.1055/s-0042-100506

37. Reissig A, Copetti R, Mathis G et al (2012) Lung ultrasound in the diagnosis and followup of community-acquired pneumonia. Chest 142(4):965-972. https://doi.org/10.1378/chest. 12-0364

38. Volpicelli G, Elbarbary M, Blaivas M et al (2012) International evidence-based recommendations for point-of-care lung ultrasound. Intensive Care Med 38(4):577-591. https://doi.org/10.1007/ s00134-012-2513-4 
39. Soldati G, Smargiassi A, Inchingolo R et al (2020) Proposal for international standardization of the use of lung ultrasound for patients with COVID-19. J Ultrasound Med. https://doi.org/10.1002/jum. 15285

40. Stone M (2020) COVID-19 Lung Ultrasound Triage. https://www.butterflynetwork.com/covid19/ role-of-pocus. Zugegriffen: 14. Mai 2020

41. AWMF Leitlinien-Register Nr. 065/002 Diagnostik und Therapie der Venenthrombose und Lungenembolie 2015. https://www.awmf. org/uploads/tx_szleitlinien/065-002I_S2k_VTE 2016-01.pdf.Zugegriffen: 17. Mai 2020

42. Wong HYF, Lam HYS, Fong AH-T et al (2019) Frequency and distribution of chest radiographic findings in COVID-19 positive patients. Radiology. https://doi.org/10.1148/radiol.2020201160

43. Wu J, Pan J, Teng D et al (2020) Interpretation of CT signs of 2019 novel coronavirus (COVID-19) pneumonia. Eur Radiol. https://doi.org/10.1007/ s00330-020-06915-5

44. Inui S, Fujikawa A, Jitsu M et al (2020) Chest CT findings in cases from the cruise ship "diamond princess" with Coronavirus disease 2019 (COVID19). Radiology 2(2):e200110. https://doi.org/10. 1148/ryct.2020200110

45. Caruso D, Zerunian M, Polici M et al (2020) Chest CT Features of COVID-19 in Rome, Italy. Radiology. https://doi.org/10.1148/radiol.2020201237

46. Hu Q, Guan H, Sun Z et al (2020) Early CT features and temporal lung changes in COVID19 pneumonia in Wuhan, China. Eur J Radiol 128:109017. https://doi.org/10.1016/j.ejrad.2020. 109017

47. Zhang J, Zhou L, Yang Yetal (2020) Therapeutic and triage strategies for 2019 novel coronavirus disease in fever clinics. Lancet Respir Med 8(3):e11-e12. https://doi.org/10.1016/S2213-2600(20)30071-0

48. Ai T, Yang Z, Hou Het al (2020) Correlation of Chest CT and RT-PCR testing in Coronavirus disease 2019 (COVID-19) in China. Radiology. https://doi.org/10 1148/radiol.2020200642

49. Fang $\mathrm{Y}$, Zhang $\mathrm{H}$, Xie J et al (2020) Sensitivity of Chest CT for COVID-19. Radiology. https://doi.org/ 10.1148/radiol.2020200432

50. Hope MD, Raptis CA, Shah A et al (2020) A role for CT in COVID-19? What data really tell us so far. Lancet 395(10231):1189-1190. https://doi.org/10.1016/ S0140-6736(20)30728-5

51. Bernheim A, Mei X, Huang M et al (2020) Chest CT findings in Coronavirus disease-19 (COVID19). Radiology. https://doi.org/10.1148/radiol. 2020200463

52. Isikbay M, Hope MD, Raptis CA et al (2020) CT on the diamond princess. Radiol Cardiothorac Imaging 2(2):e200155. https://doi.org/10.1148/ ryct.2020200155

53. Guan W-J, Ni Z-Y, Hu Y et al (2020) Clinical Characteristics of Coronavirus Disease 2019 in China. N Engl J Med 382(18):1708-1720. https:// doi.org/10.1056/NEJMoa2002032

54. Li Y, Xia L (2020) Coronavirus Disease 2019 (COVID19). AJR Am J Roentgenol. https://doi.org/10. 2214/AJR.20.22954

55. Lohöfer FK, Makowski MR, Kaissis GA et al (2020) CT-Diagnostik bei COVID-19: Nutzen und Limitationen im klinischen Alltag. Dtsch Arztebl 117(17):A-876

56. American College of Radiology (ACR) (2020) ACR Recommendations for the use of Chest Radiography and Computed Tomography (CT) for Suspected COVID-19 Infection. https:// www.acr.org/Advocacy-and-Economics/ACRPosition-Statements/Recommendations-for-
Chest-Radiography-and-CT-for-SuspectedCOVID19-Infection. Zugegriffen: 13. Mai 2020

57. Canadian Society of Thoracic Radiology Canadian Society of Thoracic Radiology and the Canadian Association of Radiologists' Statement on COVID19. https://car.ca/news/canadian-society-ofthoracic-radiology-and-canadian-associationof-radiologists-statement-on-covid-19/. Zugriff am 13. Mai 2020

58. Deutsche Röntgengesellschaft Information der AG Thoraxdiagnostik der Deutschen Röntgengesellschaft. https://www.drg.de/de-DE/5995/covid19/.Zugriffam 13. Mai 2020

59. Wen Z, Chi Y, Zhang L et al (2020) Coronavirus disease 2019. Radiol Cardiothorac Imag ing 2(2):e200092. https://doi.org/10.1148/ryct. 2020200092

60. Rubin GD, Ryerson CJ, Haramati LB et al (2020) The role of chest imaging in patient management during the COVID-19 pandemic. Radiology. https:// doi.org/10.1148/radiol.2020201365

61. Wang YXJ, Liu W-H, Yang M et al (2020) The role of CT for Covid-19 patient's management remains poorly defined. Ann Transl Med 8(4):145. https:// doi.org/10.21037/atm.2020.02.71

62. Dommasch M, Spinner CD (2020) Triagestrategie für COVID-19-Verdachtsfälle bei steigender Patientenzahl. NotfRettMed.https://doi.org/10.1007/ s10049-020-00700-z

63. Osterwalder J (2020) COVID-19-mehr Lungen-PoCUS und sparsam mit Stethoskop, Thoraxröntgen und Lungen-CT umgehen. Praxis. https://doi.org/ 10.1024/1661-8157/a003512

64. Stock K (2020) Registerstudie „Rolle des bettseitig durchgeführten Lungenultraschalls bei Patienten mit COVID-19-Infektion zur Diagnostik, Therapiekontrolle und als Prognostikum" (Votum der Ethikkommission TU München 266/20S.

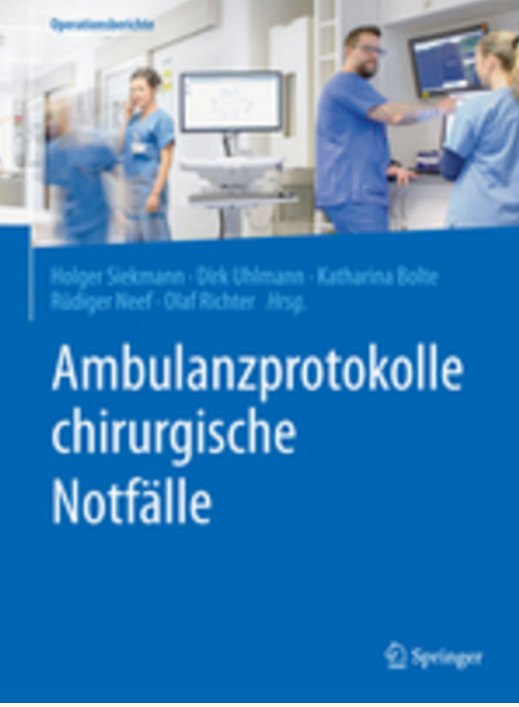

Über 150 Beispiele für die einwandfreie Abfassung von Berichten in einer Notfallambulanz

H. Siekmann, D. Uhlmann, K. Bolte, R. Neef, O. Richter (Hrsg)

Das Verfassen eines formal und inhaltlich korrekten und rechtlich einwandfreien Berichts in einer Notfallambulanz ist in diesem Buch an über 150 beispielhaften Berichten aus der Unfallchirurgie und Orthopädie sowie der Allgemein-, Viszeral-, Gefäß- und Thoraxchirurgie anschaulich dargestellt. Sowohl Berichte zur stationären Aufnahme wie auch solche zur ambulanten Weiterbehandlung haben Platz gefunden. Einführende Kapitel erläutern den systematischen Berichtsaufbau und die rechtlichen Hintergründe sowie fachspezifische Besonderheiten. Zudem bieten die Berichte durch ihre Realitätsnähe vor allem dem Chirurgen in der Weiterbildung die Möglichkeit, sich auf seine Tätigkeit in der Ambulanz vorzubereiten.

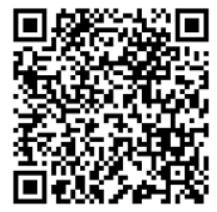

QR-Code zum Buch 